\title{
ANALISIS COMPORTAMENTAL DE LAS LEYES DE NEWTON
}

MIGUEL, 0.

Instituto de Matemática Aplicada San Luis

Universidad Nacional de San Luis

\section{SUMMARY}

The aim of this paper is to present a didactic view of Newton's lows with particular emphasis on the analysis of the most usual sources of error in their understanding.

\section{INTRODUCCION}

Identificar o aprender un concepto consiste en generalizar entre la clase de ejemplos del mismo y en discriminar entre ejemplos y no-ejemplos. (Miguel, 1981).

La identificación de un concepto se realiza sometiendo al alumno a estímulos: ejemplos o pares ejemplos contraejemplos, hasta que logra determinar los atributos comunes a todos los ejemplos: estos atributos son relevantes (AR) a la captación del concepto. Los demás atributos no son relevantes al mismo. Entre estos últimos, hay algunos que, por ser muy llamativos, pueden fijarse en la mente del alumno como si fuesen relevantes: Los Ilamaremos atributos irrelevantes (AI). Como veremos, una parte importante del proceso de aprendizaje consiste en evitar la fijación de los AIs.

La definición de un concepto es la enumeración de sus ARs.

Los ejemplos de un concepto contienen todos sus ARs y pueden contener o no AIs. Un no-ejemplo o contraejemplo del mismo carece de, por lo menos, un AR.

Llamaremos «afirmación conceptual» (AC) a toda frase que relacione conceptos. Entre las ACs están las leyes y los principios. Las ACs se tratan igual que los conceptos, pues tienen ARs y AIs. Para aprender una AC tienen que haberse fijado previamente los ARs de Ios conceptos que ella relaciona. En general, la frase que constituye la $A C$ describe el único $A R$ que ella posee.

Las tres leyes de Newton, tal como son definidas en los textos, son ejemplos de ACs.
El objeto de este trabajo es dar una visión pedagógica de las leyes mencionadas. No intentamos filosofar acerca de su validez en ciertos campos o de la pertinencia o no de sus enunciados. Es posible que el punto de vis. ta didáctico coincida con el filosófico y ello será una afirmación de la unidad de la física.

\section{UN VISTAZO A LOS PREREQUISITOS}

Las dos primeras leyes se refieren a acciones (o no) de las fuerzas sobre la velocidad de un cuerpo, mientras que la tercera se refiere a las características de la interacción (fuerzas) entre dos cuerpos.

Para comprender las leyes, el alumno debe tener claros los conceptos de velocidad constante y de cambios en la velocidad. Los AR de «velocidad constante» son: 1: constancia en el módulo. 2: Constancia en la dirección y 3: Constancia en el sentido. Para reposar los ARs de estos conceptos son útiles los ejemplos del tipo siguiente: Caso 1: Un cuerpo en movimiento rectilineo pero no uniforme (dirección y sentido constantes, módulo variable). Caso 2: cuerpo en movimiento circular uniforme (magnitud constante, dirección variable, sen. tido constante?). Caso 3: cuerpo que incide perpendicularmente contra una pared y rebota elásticamente en la misma dirección (magnitud y dirección constantes, sentido variable).

En cada uno de estos casos falta un AR del concepto "velocidad constante" y son buenos contraejemplos para el mismo. En el caso de "cambios en la velocidad" ejemplifican cada una de las posibilidades. 
El alumno debe manejar también los conceptos de fuer$z a$, sistema de fuerzas y fuerza resultante. Por lo tanto debe conocer alguna de las reglas para sumar vectores fuerza.

En realidad, la definición de fuerza surge de la $2 a$. ley como "aquello capaz de producir aceleraciones» o de la 3a. ley como las «interacciones entre cuerpos». La introducción del concepto de fuerza previamente al estudio de las leyes de Newton pueden dar origen a falsas concepciones, tal como veremos más adelante.

\section{LA PRIMERA LEY DE NEWTON}

Podemos enunciarla así: «Si la fuerza resultante que actúa sobre un cuerpo es nula, permanecerá a velocidad constante (en un sistema de referencia inercial)". Más precisamente:

$$
\Sigma \vec{F}=0 \rightarrow \vec{v}=\text { cte } \rightarrow \vec{a}=0
$$

Los prerequisitos de la ley son los conceptos «velocidad constante», con su caso particular $\vec{v}=0$ «fuerza resultante» y la relación de biimplicación, aunque no se le exprese en forma explícita: es importante señalar que la ley vale también en sentido inverso: si observamos que un cuerpo se mueve a velocidad constante, entonces podemos asegurar que la fuerza resultante so. bre él vale cero.

En la literatura pueden encontrarse enunciados de esta ley del tipo «Un cuterpo en reposo o a velocidad constante permanecerá en su estado de movimiento a menos que actúen sobre él fuerzas exteriores que lo obliguen a cambiar dicho estado». Este enunciado es coherente cont el nuestro, pues el estado de «reposo o velocidad constante» se debe a que $\Sigma \overrightarrow{\mathrm{F}}=0$.

No dice lo mismo un enunciado del tipo «Un cuerpo libre de fuerzas permanece en estado de reposo o a velocidad constanter, ya que el estudiante no encontrará nunca un cuerpo libre de fuerzas. Este enunciado no puede inferirse de la experiencia. No discutiremos este enunciado, pero sí reconocemos que es muy útil para eliminar la idea preconcebida de que son necesarias fuerzas para mantener a los cuerpos en movimiento.

Un buen contraejemplo para esta idea es la de un ciclista que deja de pedalear. La bicicleta seguirá moviéndose a casi la misma velocidad que trafa.

Atributos de la primera ley.

El enunciado relaciona los conceptos de fuerza resultante con velocidad constante y este es el único AR.

\section{Algunos AI importantes son:}

1) Es irrelevante que se trate de un caso estático ( $\vec{v}=$ 0) o dinámico $(\mathrm{v}=$ cte.). Este Al es llamativo por la estructura de los libros de texto que separan los casos antedichos.
2) Si aceptamos el primer enunciado, es irrelevante que el cuerpo esté en un caso real «libre de fuerzas» o en el caso «resultante nula». En realidad ambos casos son indistinguibles, ya que el criterio que se utiliza para decidir si un cuerpo está o no libre de fuerzas es que viaje o no a velocidad constante. Este AI resulta llamativo por los ejemplos dados en casi todos los textos.

Encontrados los ARs y los principales AIs, el profesor debe preparar ejemplos y contraejemplos de los mismos. Deben tenerse en cuenta dos cosas importantes: primero, los ejemplos no deben repetir el mismo AI para evitar su fijación, segundo, el uso de contraejemplos debe ser muy cuidadoso, porque la memoria tiende finalmente a confundir las cosas y puede ocurrir que se recuerde el contraejemplo como un ejemplo.

En los textos aparecen una serie de experimentos en los cuales se hace deslizar un bloque sobre superficies cada vez más lisas de modo a ir eliminando la fricción. Luego se extrapola al caso en que la fricción es nula. Desde nuestro punto de vista, lo que se hace es presentar una sucesión de contraejemplos en los que el AR va alcanzando de a poco su existencia. Estas series de contraejemplos son realmente valiosas para favorecer la discriminación entre casos límites en los que el AR pasa de "no estar» a "estar». Recomendamos su uso. En este caso, un cuerpo sobre colchón de aire constituye el mejor caso límite.

\section{LA SEGUNDA LEY DE NEWTON}

El enunciado con que trabajamos es «la fuerza resultante ejercida sobre un cuerpo es igual al producto de su masa (inercial) por la aceleración que la fuerza le producen. En símbolos:

$$
\mathbf{\Sigma} \overrightarrow{\mathrm{F}}=\mathrm{m} \overrightarrow{\mathrm{a}} \text {. }
$$

Los prerequisitos de esta ley son los de fuerza resultante y de aceleración. Los ejemplos de aceleración son los contraejemplos de "velocidad constante» de los casos 1 a 3 y viceversa. Debe ponerse especial atención al caso en que la aceleración implica un cambio en la dirección (ejemplifique más) y a los casos en que haya velocidad nula pero aceleración, como pueden ser:

Caso 4: Piedra arrojada hacia arriba en el momento de pasar por el punto más alto.

Caso 5: Péndulo o sistemas resorte-masa en el extremo de sus oscilación.

Para su mejor comprensión, puede separarse la ley en dos ACs:

AC1: «Para un cuerpo dado, $(m=$ cte) la aceleración es proporcional a la fuerza resultantes.

AC2: «Pára una fuerza resultante dada, actuando sobre distintos cuerpos, las aceleraciones producidas serán proporcionales a las inversas de sus masas inerciales». 
Analicemos la AC1. Dados los ARs de los prerequisitos como conocidos, el único $\mathrm{AR}$ de la $\mathrm{AC}$ es ella misma.

Puede comenzarse con ejemplos muy simples, que muestren los efectos de la fuerza sobre el módulo de $\vec{v}$ del tipo:

1) Sobre un cuerpo dado actúa una fuerza y le produce una aceleración de $10 \mathrm{~m} / \mathrm{seg} .2$. ¿Qué aceleración le producirán al mismo cuerpo? $2 \mathrm{~F}, 3 \mathrm{~F}, 10 \mathrm{~F}$ ?

2) Experimentalmente, medir las aceleraciones producidas sobre un carrito por $1 \mathrm{~F}, 2 \mathrm{~F}$, etc. mediante el uso de 1. 2. etc. resortes o gomitas iguales.

Yendo luego a ejemplos más complicados. Mostrar ejemplos en los que F cambia la dirección (Caso 2) o el sentido (Caso 3) de la velocidad para mostrar que $F$ tiene igual dirección y sentido que la aceleración.

Los contraejemplos ayudan a fijar la AC y serán los ejemplos de la primera ley.

Observación: Si aceptamos el primer enunciado de la 1a. ley, ella puede considerarse como un caso particular de esta AC. Este es el mejor momento para introducir el concepto de compensar fuerzas: Por ejemplo: un carrito que desciende un plano inclinado de tal modo que su velocidad es constante debido a la acción conjunta de la fricción y de la componente del peso.

Observacion: Suele utilizarse esta AC para definir fuerza como «aquello (lo único) capaz de producir aceleraciones».

Ahora veamos la $\mathrm{AC2}$ :

El único AR de la misma es la relación que expresa. También se ejemplifica de lo simple a lo complejo comenzando por algo as:

1) Una cierta $F$ al actuar sobre una masa $m$ le produce una aceleración de $90 \mathrm{~m} / \mathrm{seg} .2$. ¿Qué aceleraciones producirá la misma F sobre masas $2 \mathrm{~m}, 3 \mathrm{~m}, 10 \mathrm{~m}$ ?

2) Experimento similar al anterior, pero tirando con un resorte dado un carrito, dos carritos, etc. y midiendo las aceleraciones.

Masa inercial: Este concepto se introduce a partir de la AC2 como «la propiedad de los cuerpos de presentar resistencia a ser acelerados».

El único AR es dicha propiedad, que se pone de manifiesto bajo la acción de una fuerza. Los ejemplos son similares a los de la AC2. Debe evitarse aquí la pesada de los cuerpos, para no introducir el Al que se mencionará.

Los principales AIs son el volumen, el tipo de material y la confusión de masa (inercial) con peso (proveniente de la masa gravitatoria). Algunos contraejem. plos típicos de los AIs: Cajas de distinto tamafio pero rellenas de modo que tengan igual masa, (lo cual se manifiesta tratando de acelerarlas). Cubos del mismo as- pecto y color, pero de distinto material. Hacer que el alumno sostenga un cuerpo (m gravitatoria) y luego to sacuda horizontalmente ( $m$ inercial).

\section{TERCERA LEY DE NEWTON}

Usaremos el siguiente enunciado: "Cuando dos cuerpos interaccionan, la fuerza que el primer cuerpo ejerce sobre el segundo (acción) es igual y opuesta a la fuer. za que el segundo ejerce sobre el primero (reacción)"). En símbolos:

$$
\vec{F}(1.2)=\vec{F}(2.1)
$$

Los ARs son:

AR1: La fuerzas en la naturaleza existen a pares, no hay fuerzas aisladas.

AR2: Acción y reacción tienen igual módulo

AR3: Tienen igual dirección

AR4: Tienen distinto sentido

AR5: Para que hayan fuerzas deben existir al menos dos cuerpos. Acción y reacción actúan sobre cuerpos distintos.

Los principales AIs son:

AIl: Es irrelevante que la interacción sea «de contacto» o «a distancia»

AI2: Son irrelevantes los valores de las masas que interactúan

Al3: Es irrelevante que el caso sea estático o dinámico.

Los ejemplos, de lo simple a lo complejo, pueden ser:

Caso 6: La tierra ejerce la fuerza peso $\overrightarrow{\mathrm{P}}$ sobre un cuer * po. El cuerpo ejerce la fuerza $\overrightarrow{\mathrm{P}}$ sobre la tierra.

Caso 7: El caso 6, pero el cuerpo está apoyado sobre una mesa. Aparece la fuerza $\vec{N}$ y su reacción $\vec{N}$.

Caso 8: Un cuerpo colgado de un resorte y éste del techo.

Analicemos brevemente los AIs.

All: Desde el punto de vista didáctico podemos divi. dir a las interacciones en dos tipos: "a distancia» de las cuales la más conocida por los estudiantes es la gravitatoria, e interacciones «de contacto», que necesitan, precisamente el contacto entre los cuerpos que interaccionan. Comprender esto último es muy importante pa* ra evitar falsas concepciones. Los casos ejemplifican juntos los dos tipos de interacciones, para evitar este Al.

Al2: En el caso 6, una pregunta típica de los alumnos es: Si el cuerpo tira de la tierra hacia arriba. ¿Por qué la tierra no sube? La tierra tiene masa infinitamente mayor que el cuerpo, por lo tanto su aceleración será infinitamente pequefia. Los cuerpos fijados a la tierra tienen masa infinita respecto a los cuerpos sueltos.

A13: Este AI es llamativo por la estructura de los tibros de texto que separan los casos estático y dinámi- 
co, pudiendo hacer pensar a los alumnos que son casos diferentes de interacciones.

\section{LAS PRECONCEPCIONES ACERCA DE LAS LEYES DE NEWTON}

El estudio de las preconcepciones de los alumnos es importante dado que ciertas teorfas del aprendizaje afirman que el sujeto que aprende busca adecuar los nuevos conocimientos a las estructuras conceptuales ya conocidas por él. Así puede ocurrir que lo nuevo no armonice con lo anterior, en tal caso, el alumno puede rechazar lo que se intenta enseñar o directamente ignorarlo.

En el tema que nos ocupa, las leyes de Newton, hay falsas concepciones muy fuertes, capaces de perdurar Iuego de completado el curso. A continuación haremos un breve relato de las principales de ellas, recurriendo a lo explorado en varios palses (McDermott, 1983). Los testimonios son coincidentes y no difieren de lo observado en muestros cursos.

\section{1) La fuerza «viva» y la fuerza «inerte».}

Consiste en asociar la posibilidad de ejercer fuerzas a algo activo (personas, animales, motores). Esta forma de pensar es muy resistente a la instrucción. De acuerdo con ella, los objetos inmóviles no ejercen fuerzas. En una encuesta realizada sobre un bloque en reposo, el $50 \%$ de los alumnos omitió la fuerza normal. Otras investigaciones muestran resultados similares. Esta falsa concepción es alentada por los textos que introducen la fuerza como un esfuerzo muscular.

2) Las fuerzas que «traen» los objetos en movimiento.

Estas fuerzas son un reflejo de la concepción pregalileana de la inercia. Las investigaciones coinciden en que los sujetos inventan una fuerza que mueve a los objetos y que tiene extraftas propiedades. Un resultado típico es el siguiente: (Clement, 1982).

El movimiento continuo, aun a velocidad constante, es debido a una fuerza que lo produce.

Si hay fuerzas opuestas que son evidentes, el movimiento se explica porque la fuerza inventada es mayor.

La fuerza inventada tiene la propiedad de crecer o disminuir para explicar los cambios en el movimiento (a mayor fuerza, más velocidad). También cambia su dirección para explicar las mismas variaciones en la velocidad. O sea, $\vec{F}$ inventada proporcional a $\vec{v}$.

3) Las «reacciones» de vinculo.

La comprensión de la tercera ley tropieza a menudo con el escollo del nombre asignado a las fuerzas de vínculo. Muchos textos las llaman aún reacciones de vinculo, y como la palabra reacción está asociada a una de las fuerzas en las interacciones, el término confunde.

\section{4) La «fuerza centrífuga»}

Esta fuerza ficticia puede definirse en un sistema de referencia no inercial en rotación para explicar la aparente contradicción con las leyes de la dinánica. $\operatorname{Sin}$ embargo, los estudiantes la confunden con una fuerza real que tira a los objetos hacia afuera en los sistemas que rotan. La confusión es alentada por los nombres dados en la vida real, por ejemplo, el artefacto doméstico que centrifuga la ropa.

\section{EL USO DE EJEMPLOS Y CONTRA- EIEMPLOS AL EVALUAR}

Al evaluar el aprendizaje de un concepto, debemos estar seguros de que el alumno fijó todos los ARs del mismo y no fijó ningún AI. Para verificar esto, conviene enfrentar al alumno con ejemplos y contraejemplos del concepto. El alumno debe aceptar los ejemplos y rechazar (decir que no son ejemplos) los contraejemplos (Miguel, 1985). Pensemos que el alumno compara "su» concepto con el ejemplo, o sea se fija si están en él todos los atributos que él cree que debe poseer, o sea «sus» ARs.

Los ejemplos tienen todos los ARs del concepto, por lo tanto el alumno encontrará todos sus ARs. Si en «SU» concepto falta algún AR, pensará que el AR del concepto es un AI de los que siempre existen. De aquf que no se pueden usar ejemplos para detectar la falta de algún $\mathrm{AR}$.

Sin embargo, pueden usarse contraejemplos en los que falta el AR que queremos evaluar. Si el alumno acepta el contraejemplo, o sea lo confunde con un ejemplo, es que no fijó tal AR.

Tenemos el caso de la la. ley. Para evaluar el concepto "velocidad constante» usamos la serie de casos 1 , 2 y 3.

Si acepta el caso 1 como ejemplo, es que no entendió que debe ser constante el módulo. Si acepta el caso 2 , no fijó que debe ser constante la dirección y si acepta el caso 3 no fijó la constancia del sentido.

Para evaluar los AIs se usan ejemplos que contengan todos los Als menos el que se quiere evaluar. EI rechazo del ejemplo indica la fijación del AI.

En la 3a. ley: si presentamos un ejemplo del caso dinámico y no lo acepta, es porque fijo que la ley vale solo en el caso estático (AI3). Si usamos una masa pequeña y otra grande y no lo acepta es porque no fijó la independencia de la ley respecto a las masas. Si no acepta un ejemplo con fuerzas de contacto es porque cree que la ley vale para acciones a distancia (AI1).

\section{CONCLUSIONES}

De los análisis anteriores se puede observar que hay tres fuentes importantes de errores conceptuales al apren- 
der las leyes de Newton. Ellos son:

1) Las preconcepciones de los estudiantes, que en general están fijadas muy fuertemente y provienen de una errónea interpretación de la reatidad.

2) El desconocimiento de los prerequisitos. Hay confusiones llamativas en la cinemática entre velocidad y aceleración. En el caso de la fuerza resultante, no especifican todas las fuerzas o suman mal.

3) El principal error al interpretar una ley dada proviene del desconocimiento de las otras. Por ejemplo en el caso 6, no se acepta la existencia de la reacción porque no se entiende que, siendo las fuerzas iguales, a mayor masa habrá menos aceleración.

Cuando se dice que «el peso de un objeto rompió la mesa (figura 1)», el peso actúa sobre el objeto y mal puede romper la mesa. Quien rompe la mesa es $\vec{N}$, la reacción a la normal.

Cuando dicen que la fuerza que trae la piedra rompió el vidrio, $n$ entendieron la $1 \mathrm{a}$. ley porque desconocen la 3 a. El. vidrio detiene a la piedra con una fuerza $\vec{F}$.

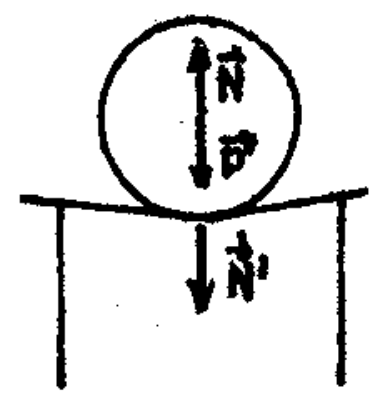

Figura 1

\section{REFERENCLAS BIBLIOGRAFICAS}

CLEMENT, J., 1982, Student's preconceptions in introductory mechanics. Am. Jour. Phys. 50, 66-71.

MC DERMOTT, L., 1983, Critical Review of Research in the Domain of Mechanics. Proc. First. Workshop of Res. on Phys. Educ. pag. 139. mientras que su reacción $\mathrm{F}$ actúa sobre el vidrio y lo rompe.

Es decir que las tres leyes son una unidad didáctica y como tal deben ensentarse. En general se comete el error de dar mucho énfasis a la 2a. ley y tratar rápidamente a la la y más aún a la 3a. Por el contrario, al final del tema deben abundar los ejemplos en que se usan las leyes simultáneamente como puede ser:

Caso 9: Figura 2: Una cura sin fricción con el piso sobre la que desliza sin fricción un bloque hacia abajo. Estudiar el movimiento de los dos cuerpos.

Esto nos lleva a apreciar la bondad de los enfoques unificadores de las tres leyes, como el de Mach, del cúal puede verse un buen tratamiento en Roederer (1966). Otro factor unificante es el hablar de interacciones entre cuerpos y no de fuerzas sobre un cuerpo.

En el transcurso de este trabajo hemos expuesto cosas conocidas por el lector de una forma diferente. Esperemos que ello haya sido útil para organizar sus clases. Esta es nuestra única aspiración y nos sentiremos contentos si así ocurre.

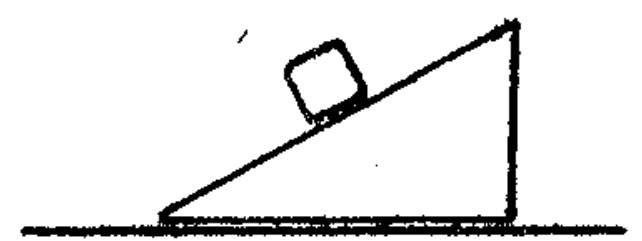

Figura 2
MIGUEL, O., 1981, Esctructuras y estrategias 6ptimas para cursos de Física, Tesis doctoral, San Luis.

MIGUEL, O., 1983, La evaluación del aprendizaje de conceptos. VI Reunion de Ed. en la Matem. Tucuman.

ROEDERER. J.G., 1966, Mecánica Elemental, EUDEBA, Bs. As. 